\title{
Instalación de FREEBSD en una máquina virtual
}

\author{
Installation of Freebsd in a Virtual Machine \\ Laura A Zapata-Hernández ${ }^{a}$, Elizabeth Hernández-Garnica ${ }^{b}$
}

\begin{abstract}
:
This practice refers to the process that must be carried out to perform the installation of the Free BSD operating system developed by UNIX based on 4.4BSD-Lite focused on Intel (x86 and Pentium), AMD64, Alpha and Sun UltraSPARC computers. This will be installed in a virtual machine with the help of the Virtual Box tool which is a virtualization software for x86 / amd64 architectures. It is currently developed by Oracle Corporation as part of its family of virtualization products. Through this application it is possible to install additional operating systems, known as "guest systems", within another "host" operating system, each with its own virtual environment.
\end{abstract}

\section{Keywords:}

Operating System, Virtualization, guest systems, host system, virtual environment, software, computer, virtual machine, installation

\section{Resumen:}

Esta práctica hace referencia al proceso que se debe llevar a cabo para realizar la instalación del sistema operativo Free BSD desarrollado por UNIX basado en 4.4BSD-Lite enfocado a computadoras Intel (x86 y Pentium), AMD64, Alpha y Sun UltraSPARC. Este se instalará en una máquina virtual con ayuda de la herramienta Virtual Box que es un software de virtualización para arquitecturas x86/amd64. Actualmente es desarrollado por Oracle Corporation como parte de su familia de productos de virtualización. Por medio de esta aplicación es posible instalar sistemas operativos adicionales, conocidos como "sistemas invitados", dentro de otro sistema operativo "anfitrión", cada uno con su propio ambiente virtual.

\section{Palabras Clave:}

Sistema Operativo, Virtualización, sistemas invitados, sistema anfitrión, ambiente virtual, software, ordenador, máquina virtual, instalación

\section{Introducción}

A medida que pasa el tiempo el avance tecnológico ha brindado a la sociedad grandes herramientas que facilitan el día a día de los seres humanos. Alrededor de los años 60 cuando se comenzó a construir computadoras, el software que se refiere a las instrucciones que permiten la interacción de los componentes físicos con los usuarios, se volvió algo indispensable. El incremento de la complejidad del hardware y los programas de aplicaciones eventualmente hicieron del sistema operativo una necesidad. Hoy en día existen sistemas operativos con licencia de paga como los de Microsoft, aunque tras la innovación y necesidades de cada usuario se ha creado un software libre que te da diferentes permisos para la manipulación y mejoramiento del mismo y como este existen los derivados de Unix como FreeBSD.

FreeBSD es un sistema operativo de UNIX basado en 4.4BSD-Lite enfocado a computadoras Intel(x86 y Pentium), AMD64, Alpha y Sun UltraSPARC. El origen de este proyecto comienza en el año de 1993, como una extensión del "Unofficial 386BSD Patchkit". David Greenman tuvo la idea de renombrar el proyecto como "FreeBSD". De las que se destacan: FreeBSD 1.0 en diciembre del año 1993, FreeBSD 1.1 en mayo del año 1994, FreeBSD en julio de 1994, FreeBSD 2.0 en noviembre de 1994, FreeBSD 2.0.5 en junio de 1995, FreeBSD 2.1.5 en agosto de 1996, FreeBSD 2.1.7.1 en febrero de 1997. En el 2012 apareció la versión 9.1 y conforme paso el tiempo de fue innovando hasta llegar a

\footnotetext{
a Alumna de Ciencias computacionales de la Universidad Autónoma del Estado de Hidalgo, Escuela Superior de Huejutla, Email: ady.d.1718@gmail.com

${ }^{\text {b }}$ Profesor por Asignatura de Ciencias Computacionales de la Universidad Autónoma del Estado de Hidalgo, Escuela Superior de Huejutla, 
la versión más actual publicada el 26 de julio del 2017, FreeBSD 11.1. (Porto, 2008)

\section{Requerimientos}

Este proyecto fue realizado en un equipo con las siguientes características:

- $\quad$ Procesador: Intel(R) Core(TM) i5-4310U CPU @ $2.00 \mathrm{GHz} 2.60 \mathrm{GHz}$

- RAM: $4.00 \mathrm{~GB}$

- $\quad$ Sistema Operativo de 64 bits, procesador x64

- Virtual box

- FreeBSD

\section{Proceso de elaboración}

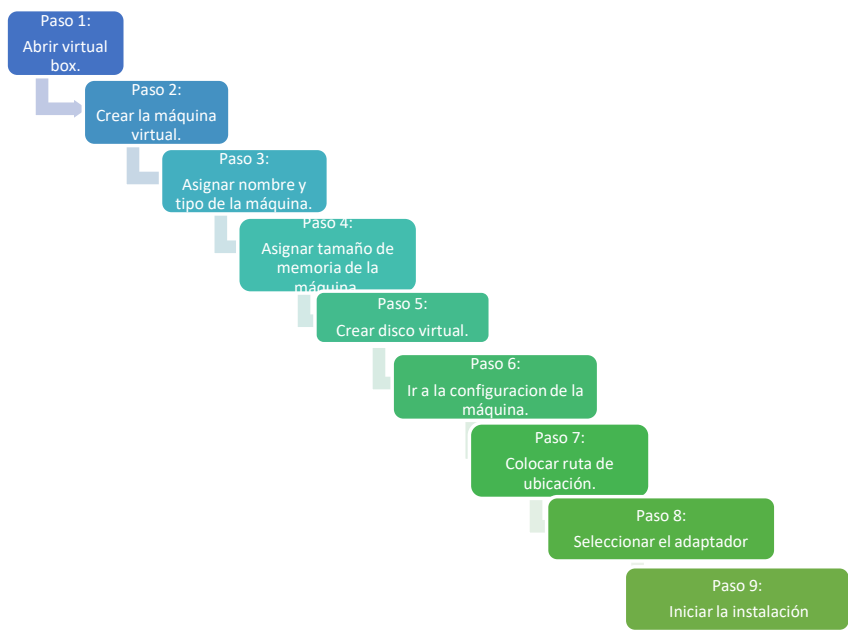

Figure 1. Proceso de instalación

Abrir virtual box dando doble clic en el icono, entrado a la aplicación en la pantalla principal seleccionar la opción

Nueva para crear la máquina virtual.

La ventana que aparece pide asignarle nombre, tipo y versión del sistema a instalar en este caso se llama FreeBSD, es de tipo BSD en su versión de 32 bits.

Dar clic en "next", en el que se pide el tamaño de memoria que se debe asignar, posteriormente se creara el disco virtual y preguntara que tipo de archivo de disco duro se utilizara seleccionar "VDI".

A continuación, se reserva un tamaño fijo para el disco o se asigna que sea de tipo dinámico en este caso se dejara en "dinámico" y dar clic en el botón de crear.

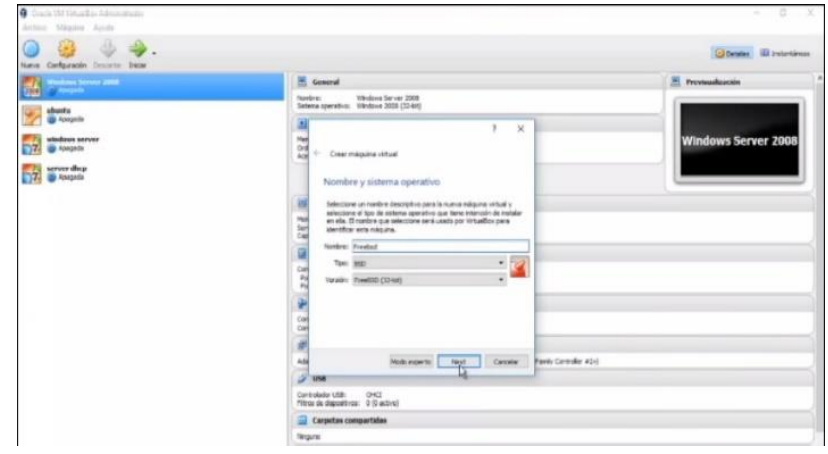

Ahora seleccionar la máquina creada, dando clic derecho sobre esta y se desplegara un menú seleccionar la configuración de la máquina.

En la pestaña "general" seleccionar la opción avanzada para colocar la ruta de ubicación de los archivos que se generaran en la instalación y posteriormente al utilizar en el sistema.

Después, ir a la pestaña "Red", y en la opción adaptador 1 seleccionar conectado a "adaptador puente", esto para tener comunicación con la máquina anfitrión.

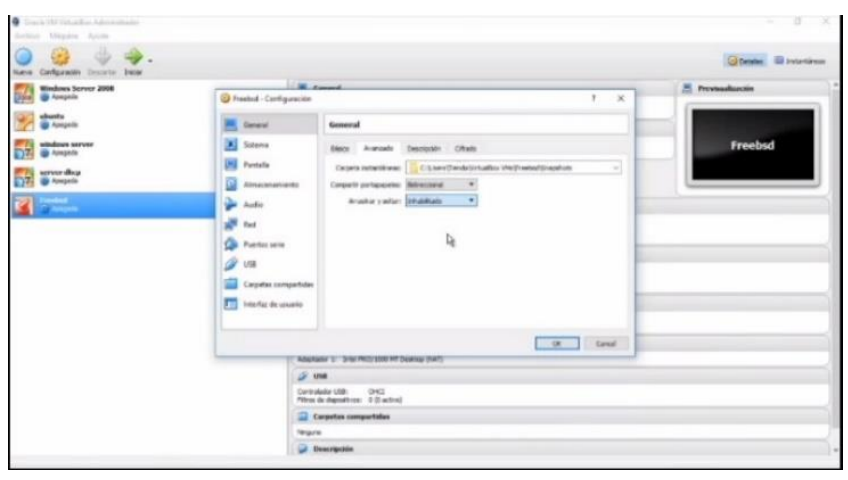

Iniciar la máquina creada en el que se pedirá la ruta donde se encuentra el archivo para la instalación, una vez seleccionado el archivo, dar enter y automáticamente va a iniciar el asistente de instalación, en el que se pedirá seleccionar el idioma que se prefiriera, así como también se deberá seleccionar el país, la hora, el nombre de usuario y contraseña a utilizar, los cuales no se deben olvidar ya que para poder acceder al sistema operativo.

Para poder utilizar el SO con cada uno de sus elementos, se deberá trabajar con ciertos comandos los cuales consisten:

\section{Comandos Utilizados en Free BSD:}

1. rm/etc/modt: Para entrar a descargar los paquetes de instalación.

2. ee/etc/resolv.conf: Para entrar a la configuración de la descarga.

3. ping -c 3 8.8.8.8: para ir a la dirección determinada.

4. portsnap fetch: Iniciar la descarga. 
5. portsnap extract: Extrae los paquetes de descarga

6. PKG install xorg: inicia la instalación de los paquetes.

7. xorg - configure pkg install gnome3: entrar a la configuración de los paquetes

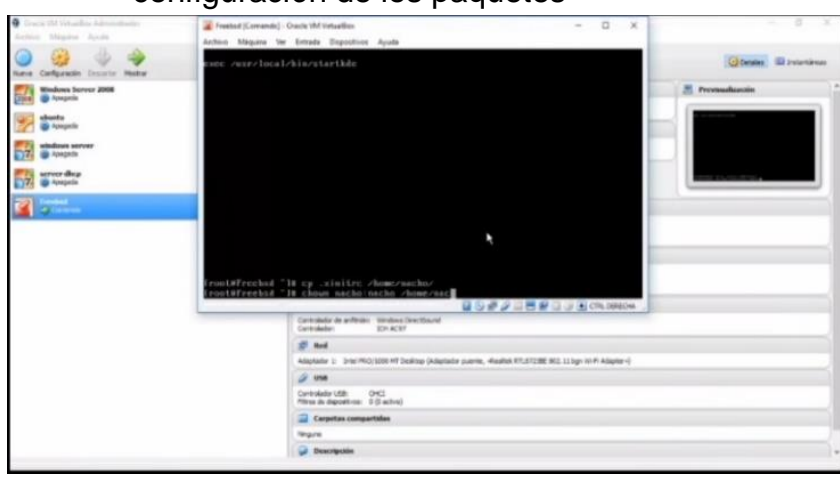

\section{Resultados obtenidos}

Pantalla principal, teclear tu login (usuario) y password (contraseña), que se establecen en el proceso de instalación, para garantizar tu entrada al sistema.

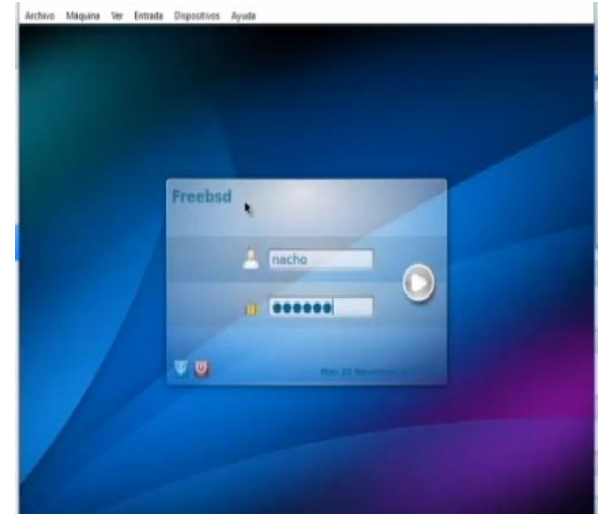

Interfaz que muestra al entrar después de introducir usuario y contraseña, la barra de tareas te aparece en la parte superior de la pantalla y sus menús son desplegables.

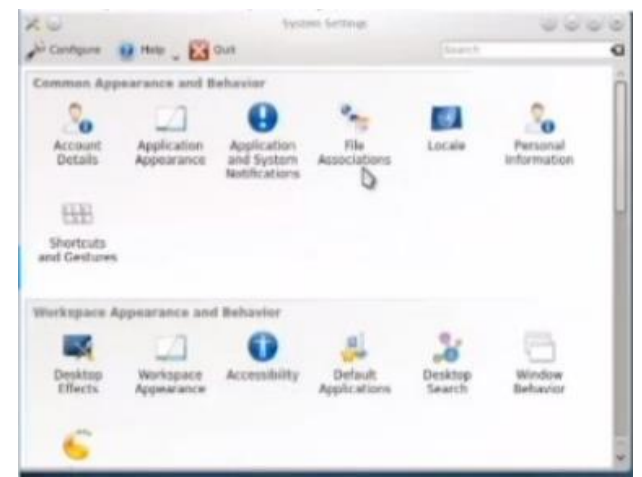

\section{Conclusión}

Con el fin de conocer los diversos sistemas operativos en su gama libre de la familia de sistemas operativos de UNIX, se instaló el FreeBSD para el conocimiento del entorno, el uso y manipulación de archivos, dispositivos de entrada/salida, seguridad y protección, además que aporta información un poco más reciente acerca de este tipo de softwares. El realizar la instalación en el entorno virtual, tiene como ventaja el no afectar la arquitectura de tu computadora, darte la experiencia de trabajar en un sistema operativo tal como lo haces realmente, solo que sin afectar a tu computadora real.

Se decidió instalar FreeBSD ya que se considera uno de los sistemas más completos y por lo tanto algo complejo de la rama libre perteneciente a la familia Unix y requería de especial investigación para poder llevarla a cabo. El conocer, usar y manipular sistemas operativos de esta rama, nos da mayores ventajas en cuanto a conocimiento de este tipo de software.

\section{Referencias}

[1]Amaya, G. (22 de abril de 2017). Manejo de memoria FreeBSD. Obtenido de https://es.slideshare.net/gamaya/manejo-dememoria-freebsd

[2]Porto, J. P. (2008). Sistema opertaivo. Obtenido de https://definicion.de/sistema-operativo/

[3]Ridcharson, A. (2017). The FreeBSD. Obtenido de https://www.freebsd.org/ 